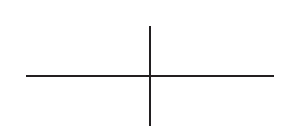

Tohoku Math. J.

56 (2004), 583-592

\title{
UN RÉSULTAT D'IRRÉDUCTIBILITÉ EN CARACTÉRISTIQUE NON NULLE
}

\author{
AleXANDRU I. BADUlescu
}

(Received April 14, 2003, revised September 12, 2003)

\begin{abstract}
Deligne, Kazhdan and Vignéras proved that, for an inner form of $G L_{n}$ over a zero characteristic $p$-adic field, the induced representation from a square integrable irreducible representation is irrreducible. Here we prove the case of non-zero characteristic.
\end{abstract}

1. Le théorème. Soient $F$ un corps local non archimédien de caractéristique quelconque, $D$ une algèbre à division centrale sur $F$, de dimension finie $d^{2}, r$ un entier strictement positif et $G=G L_{r}(D)$. Le but de ce papier est de montrer le théorème 1.1 plus bas quand le corps de base $F$ est de caractéristique non nulle. Le cas de caractéristique nulle a été prouvé dans [DKV] (Théorème B.2.d) et c'est là qu'apparaît l'idée d'utiliser le théorème de Paley-Wiener. Leur preuve ne marche pas directement en caractéristique positive. On prouve ici le cas de caractéristique non nulle, en utilisant la méthode des corps proches de Kazhdan.

THÉorème 1.1. Soit $P$ un sous-groupe parabolique de $G$ et soit $P=L U$ une décomposition de Levi de P. Soit $\pi$ une représentation de carré intégrable de L. Alors $\operatorname{ind}_{P}^{G} \pi$ est irréductible.

L'importance de ce théorème vient de ce qu'il permet de passer de la classification de Langlands à une classification plus fine, comme l'a montré Zelevinski dans [Ze] pour $G L_{n}$ et, à sa suite, Tadić dans [Ta] pour les formes intérieures. Dans [Ta], l'auteur se place en caractéristique nulle et le résultat que nous prouvons ici permet de lever cette contrainte.

2. Notations et conventions. On note $\Psi(G)$ l'ensemble des caractères lisses non ramifiés de $G$. L'ensemble $\Psi(G)$ admet une structure naturelle de variété algébrique. Si $\pi$ est une représentation admissible de $G$, on note $\Psi(G ; \pi)$ l'ensemble de classes de représentations du type $\psi \otimes \pi, \psi \in \Psi(G)(\Psi(G ; \pi)$ a une structure de variété algébrique isomorphe à un quotient de la variété $\Psi(G)$ ). On note $\operatorname{Grot}(G)$ le groupe de Grothendieck des représentations lisses de longueur finie de $G$. $\operatorname{Grot}(G)$ admet deux $Z$-bases remarquables, l'une formée des classes des représentations irréductibles, l'autre formée des classes des représentations de Langlands (voir les paragraphes suivants). Par la suite, nous regarderons une représentation de $G$ comme un élément de $\operatorname{Grot}(G)$ quand aucune confusion n'est possible.

Un sous-groupe parabolique de $G$ est dit standard s'il contient le groupe des matrices triangulaires supérieures, et un sous-groupe de $G$ est dit sous-groupe de Levi standard si

2000 Mathematics. Subject Classification. Primary 20G05; Secondary 20G25, 20 G30. 


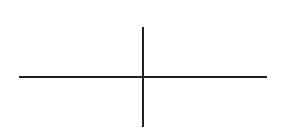

c'est la composante de Levi d'un sous-groupe parabolique standard et s'il contient le groupe des matrices diagonales. Si $L$ est un sous-groupe de Levi standard de $G$ alors il existe une partition $A_{1} \bigsqcup A_{2} \bigsqcup \cdots \bigsqcup A_{k}$ de l'ensemble $\{1,2, \ldots, r\}$ où $A_{1}=\left\{1,2, \ldots, r_{1}\right\}, A_{2}=$ $\left\{r_{1}+1, r_{1}+2, \ldots, r_{1}+r_{2}\right\}$ etc. telle que $L$ soit l'ensemble des matrices $M=\left(m_{i j}\right)_{1 \leq i, j \leq r} \in$ $G$ telles que $m_{i j}$ est nul si $(i ; j) \notin \bigcup_{u=1}^{k} A_{u} \times A_{u}$. Nous identifions alors $L$ au produit $G L_{r_{1}}(D) \times G L_{r_{2}}(D) \times \cdots \times G L_{r_{k}}(D)$. Les notations du paragraphe précédent s'appliquent facilement alors à tout sous-groupe de Levi standard de $G$.

Si $v \in \Psi(L)$, alors $v=\prod_{u=1}^{k} v_{u}$, où $v_{u} \in \Psi\left(G L_{r_{u}}(D)\right)$ et s'écrit donc $v_{u}=|\operatorname{det}|^{c_{u}}$, où $c_{u}$ est un nombre complexe. On dit que $v$ est strictement positif si $u \mapsto \operatorname{Re}\left(c_{u}\right)$ est une fonction strictement croissante.

Nous appelons représentation de Langlands une représentation du type $\operatorname{ind}_{Q}^{G} \nu \otimes \tau$ où $Q$ est un sous-groupe parabolique standard de $G, \tau$ est une représentation tempérée du sousgroupe de Levi de $Q$, et $v$ est un caractère strictement positif. L'ensemble des représentations de Langlands forme une base de $\operatorname{Grot}(G)$ ([DKV], A.4.f) que nous appellerons la base de Langlands. Nous appellerons représentation de Langlands strictement induite une représentation comme plus haut avec $Q \neq G$. On note $W(G)$ le groupe formé par les matrices de permutation dans $G$ qu'on identifie aussi avec le groupe des permutations de l'ensemble $\{1,2, \ldots, r\}$. Si $L$ est un sous-groupe de Levi standard de $G$ et $g \in G$, alors on pose ${ }^{g} L=g L g^{-1}$ (même notation aussi pour les paraboliques) et si $\pi$ est une représentation de $L$, on note ${ }^{g} \pi$ la représentation de ${ }^{g} L$ définie par ${ }^{g} \pi(x)=\pi\left(g^{-1} x g\right)$. On utilise aussi les notations: $L^{g}$ pour ${ }^{-1} L$ et $\pi^{g}$ pour $g^{-1} \pi$.

Une représentation de $G$ est dite essentiellement de carré intégrable si elle peut s'écrire comme produit tensoriel d'une représentation de carré intégrable et d'un caractère de $G$. Une représentation est dite essentiellement tempérée si elle peut s'écrire comme produit tensoriel d'une représentation tempérée et d'un caractère de $G$. Ces définitions s'étendent de façon évidente aux sous-groupes de Levi standard de $G$.

\section{La preuve.}

DÉmonstration du ThÉORÈme 1.1. On peut supposer que $P$ est standard. Nous montrons le théorème par récurrence sur l'entier strictement positif $k$ tel que $G=G L_{k}(D)$ ( $D$ est fixée). Pour $k=1$ le théorème est évident. Supposons que le théorème est vérifié pour tout $k<r$. Supposons maintenant par l'absurde qu'il existe un sous-groupe parabolique propre $P_{0}$ de $G=G L_{r}(D)$, une décomposition de Levi $P_{0}=L_{0} U_{0}$ de $P_{0}$ et une représentation de carré intégrable $\pi_{0}$ de $L_{0}$ telle que l'induite de $P_{0}$ à $G$ de la représentation $\pi_{0}$ ne soit pas irréductible. On sait qu'on a dans $\operatorname{Grot}(G)$ :

$$
\operatorname{ind}_{P_{0}}^{G} \pi_{0}=\sum_{i=1}^{s} a_{i} \tau_{i}
$$

où les $a_{i}$ sont des entiers strictement positifs et les $\tau_{i}$ sont des représentations tempérées de $G$ non équivalentes, et aucune parmi les $\tau_{i}$ n'est de carré intégrable. 


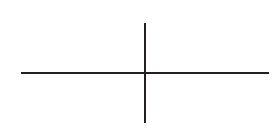

IRRÉDUCTIBILITÉ EN CARACTÉRISTIQUE NON NULLE

LEMME 3.1. On a $s=1$.

DÉmONSTRATION. Supposons par l'absurde que $s \geq 2$. On a alors deux cas:

Premier cas: Il existe un $i \in\{2,3, \ldots, s\}$ tel que $\tau_{i} \notin \Psi\left(G ; \tau_{1}\right)$. On peut supposer que $i=2$. Posons

$$
\left\{\tau_{1}, \tau_{2}, \tau_{3}, \ldots, \tau_{s}\right\} \cap \Psi\left(G ; \tau_{1}\right)=\left\{\tau_{i_{1}}=\tau_{1}, \tau_{i_{2}}, \tau_{i_{3}}, \ldots, \tau_{i_{p}}\right\}
$$

et

$$
\left\{\tau_{1}, \tau_{2}, \tau_{3}, \ldots, \tau_{s}\right\} \cap \Psi\left(G ; \tau_{2}\right)=\left\{\tau_{j_{1}}=\tau_{2}, \tau_{j_{2}}, \tau_{j_{3}}, \ldots, \tau_{j_{q}}\right\}
$$

Soient

$$
\alpha=\sum_{u=1}^{q} a_{j_{u}}
$$

et

$$
\beta=\sum_{v=1}^{p} a_{i_{v}} .
$$

On considère alors la forme linéaire $f$ sur $\operatorname{Grot}(G)$ définie sur la base de Langlands par:

(1) $f(\rho)=\alpha$ si $\rho \in \Psi\left(G ; \tau_{1}\right)$

(2) $f(\rho)=-\beta$ si $\rho \in \Psi\left(G ; \tau_{2}\right)$

(3) $f(\rho)=0$ si $\rho$ est une représentation de la base de Langlands de $\operatorname{Grot}(G)$ qui n'appartient ni à $\Psi\left(G ; \tau_{1}\right)$, ni à $\Psi\left(G ; \tau_{2}\right)$.

Remarque. Les conditions (1) et (2) ont été choisies de façon à ce que (1), (2) et (3) impliquent que $f\left(\chi \otimes \operatorname{ind}_{P_{0}}^{G} \pi_{0}\right)=0$ pour tout $\chi \in \Psi(G)$. On a aussi $f\left(\tau_{1}\right)=\alpha \neq 0$.

Deuxième cas: Pour tout $i \in\{2,3, \ldots, s\}$ il existe un caractère $\chi_{i} \in \Psi(G)$ tel qu'on ait $\tau_{i}=\chi_{i} \otimes \tau_{1}$. Pour une représentation tempérée donnée $x$, il existe, modulo conjugaison dans $G$, un unique couple $(L, \sigma)$ avec $L$ sous-groupe de Levi standard et $\sigma$ représentation

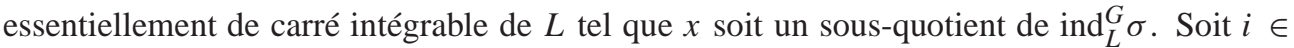
$\{2,3, \ldots, s\}$. La représentation $\tau_{i}$ est sous-quotient des l'induite à $G$ de $\left(\operatorname{res}_{P_{0}}^{G} \chi_{i}\right) \otimes \pi_{0}$, avec multiplicité $a_{1}$, et de l'induite à $G$ de $\pi_{0}$, avec multiplicité $a_{i}$. On en déduit que les $a_{i}$ sont tous égaux. Soit alors $f_{0}$ une fonction algébrique définie sur la variété $\Psi\left(G ; \tau_{1}\right)$ par: si $l$ est le plus grand entier tel que $|\operatorname{det}|^{2 \pi i / l} \otimes \tau_{1}$ est équivalente á $\tau_{1}$ alors, pour tout $\left.\alpha \in \boldsymbol{C}, f_{0}\left(|\operatorname{det}|^{\alpha} \otimes\right) \tau_{1}\right)=e^{l \alpha}$.

Définissons cette fois la fonction $f$ sur la base de Langlands de $\operatorname{Grot}(G)$ de la façon suivante:

(1') $\quad f(\rho)=f_{0}(\rho)$ si $\rho \in \Psi\left(G ; \tau_{1}\right)$

(2') $f(\rho)=0$ si $\rho$ est une représentation de la base de Langlands qui ne se trouve pas dans $\Psi\left(G ; \tau_{1}\right)$.

Remarque. Dans ce cas aussi, $f\left(\chi \otimes \operatorname{ind}_{P_{0}}^{G} \pi_{0}\right)=0$ pour tout $\chi \in \Psi(G)$, tandis que $f\left(\tau_{1}\right)=1 \neq 0$.

Nous allons montrer que la fonction $f$ vérifie toujours (qu'on soit dans le premier ou le deuxième cas) les conditions de Paley-Wiener. 


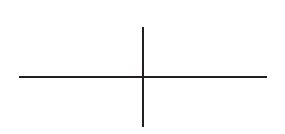

LEMME 3.2. L'application $f$ s'annule sur toute représentation induite à partir d'un sous-groupe parabolique propre de $G$.

DÉmonstration. Se fait en deux étapes:

Étape 1: $f$ s'annule sur toute induite d'une représentation essentiellement de carré intégrable d'un sous-groupe de Levi propre. (N'utilise pas l'hypothèse de récurrence.)

Soient $P$ un sous-groupe parabolique propre de $G, P=L U$ une décomposition de Levi de $P$ et $\sigma$ une représentation essentiellement de carré intégrable de $L$. On sait (voir, par exemple, [Ba1], Lemme 2.3) qu'on a deux possibilités:

- si $\sigma$ est un produit du type $\sigma=\psi \otimes \sigma_{u}$ où $\psi$ est la restriction à $L$ d'un caractère $\Psi$ de $G$ et $\sigma_{u}$ est de carré intégrable, alors ind ${ }_{L}^{G} \sigma=\Psi \otimes \operatorname{ind}_{L}^{G} \sigma_{u}$,

- sinon ind ${ }_{L}^{G} \sigma$ est une somme de représentations de Langlands strictement induites.

Maintenant, si $\sigma$ est dans la seconde situation, $f\left(\operatorname{ind}_{P}^{G} \sigma\right)=0$ par la construction de $f$ (condition (3) dans le premier cas et (2') dans le deuxième). Si $\sigma$ est dans la première situation, alors on a encore une fois deux possibilités:

- ou bien $L=L_{0}^{g}$ pour un $g \in G$ et il existe un caractère $\theta$ de $L$ qui est la restriction d'un caractère $\Theta$ non ramifié de $G$ tel que $\sigma_{u}=\theta \otimes \pi_{0}^{g}$; alors, dans $\operatorname{Grot}(G)$, on a ind ${ }_{L}^{G} \sigma_{u}=$ $\Theta \otimes \operatorname{ind}_{L_{0}}^{G} \pi_{0}$, ce qui implique

$$
f\left(\operatorname{ind}_{P}^{G} \sigma\right)=f\left(\Psi \otimes \operatorname{ind}_{L}^{G} \sigma_{u}\right)=f\left(\Psi \Theta \otimes \operatorname{ind}_{L_{0}}^{G} \pi_{0}\right)=0
$$

(voir les remarques faites plus haut sur la construction de $f$ dans les deux cas),

- ou bien on ne se trouve pas dans cette situation; alors les séries de composition de $\Theta \otimes \operatorname{ind}_{P_{0}}^{G}\left(\pi_{0}\right)$ et $\operatorname{ind}_{P}^{G} \sigma_{u}$ sont disjointes pour tout $\Theta \in \Psi(G)$. La suite de composition de ind ${ }_{P}^{G} \sigma_{u}$ est donc formée de représentations tempérées, mais ne contient aucun élément de $\bigcup_{i=1}^{s} \Psi\left(G ; \tau_{i}\right)$. Cela implique que la suite de composition de $\operatorname{ind}_{P}^{G} \sigma=\Psi \otimes \operatorname{ind}_{P}^{G} \sigma_{u}$ est formée de représentations essentiellement tempérées mais ne contient aucun élément de $\bigcup_{i=1}^{S} \Psi\left(G ; \tau_{i}\right)$. Donc, encore une fois, $f\left(\operatorname{ind}_{P}^{G} \sigma\right)=0$ par la condition (3) dans le premier cas et (2') dans le deuxième cas dans la construction de $f$.

Étape 2: Soit $\operatorname{Grot}_{\text {ind }}(G)$ le sous-groupe de $\operatorname{Grot}(G)$ engendré par toutes les représentations induites à partir de sous-groupes paraboliques propres de $G$. Les représentations induites de représentations essentiellement de carré intégrable à partir de sous-groupes paraboliques propres de $G$ forment une famille génératrice pour $\operatorname{Grot}_{\text {ind }}(G)$. (Utilise l'hypothèse de récurrence.)

Remarquons que l'hypothèse de récurrence implique que pour tout sous-groupe parabolique propre $P$ de $G$ qui a une décomposition de Levi $P=L U$, toute représentation tempérée de $L$ est une représentation induite d'une représentation de carré intégrable. Par conséquent, toute représentation essentiellement tempérée de $L$ est une représentation induite d'une représentation essentiellement de carré intégrable.

Soit $P=L U$ un sous-groupe parabolique propre de $G$. La remarque plus haut vaut maintenant pour tous les sous-groupes paraboliques de $L$, propres ou pas cette fois. Mais l'ensemble des induites des représentations essentiellement tempérées de tous les sous- 


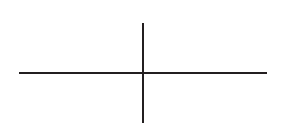

IRRÉDUCTIBILITÉ EN CARACTÉRISTIQUE NON NULLE

groupes paraboliques (propres ou pas) de $L$ est une famille génératrice de $\operatorname{Grot}(L)$ (car elle contient la base de Langlands de $L$ ). L'hypothèse de récurrence implique donc que l'ensemble des induites des représentations essentiellement de carré intégrable de tous les sous-groupes paraboliques (propres ou pas) est aussi une famille génératrice de $\operatorname{Grot}(L)$. Cela prouve que les représentations induites de représentations essentiellement de carré intégrable à partir de sous-groupes paraboliques propres de $G$ engendrent $\operatorname{Grot}_{\text {ind }}(G)$.

Pour vérifier les conditions de Paley-Wiener sur $f$, il suffit, grâce au lemme 3.2, de montrer que pour toute représentation irréductible $\pi$ de $G$, la restriction de $f$ à $\Psi(G ; \pi)$ est algébrique. Pour cela, on écrit $\pi$ sur la base de Langlands dans $\operatorname{Grot}(G)$. Il y a deux types de représentations qui apparaissent dans cette écriture: des représentations essentiellement tempérées de $G$ et des représentations de Langlands strictement induites. Quand on fait le produit tensoriel d'une représentation de Langlands strictement induite par un caractère, on obtient toujours un élément de $\operatorname{Grot}_{\text {ind }}(G)$. Or, on a montré que $f$ s'annule sur $\operatorname{Grot}_{\text {ind }}(G)$. Donc, l'algébricité de $f$ sur $\Psi(G ; \pi)$ se réduit à l'algébricité de $f$ sur les variétés $\Psi(G ; \tau)$ où $\tau$ est une représentation essentiellement tempérée, qui est évidente par les conditions posées à la construction de $f$.

Donc $f$ est une fonction trace par application du théorème de Paley-Wiener ([BDK]). Soit $f^{\prime}$ une fonction sur $G$ qui correspond à $f$ par ce théorème.

Rappelons qu'un élément de $G$ est dit semisimple régulier si son polynôme caractéristique est sans racine multiple sur une clôture algébrique de $F$, et elliptique régulier si son polynôme caractéristique est irréductible et sans racine multiple sur une clôture algébrique de $F$.

LeMme 3.3. L'intégrale orbitale de f' est nulle sur les éléments semisimples réguliers qui ne sont pas elliptiques réguliers de G.

DÉmonstration. On a vu que, pour tout $\sigma \in \operatorname{Grot}_{\text {ind }}(G)$, on avait $\operatorname{tr} \sigma\left(f^{\prime}\right)=0$. Le lemme est alors une conséquence classique en toute caractéristique (voir par exemple [Ba1], Lemme 2.4).

La fonction $f^{\prime}$ annule de plus les traces de toutes les représentations essentiellement de carré intégrable, mais pas la trace de $\tau_{1}$. Montrons qu'il y a là une contradiction qui prouve que $s=1$ (on est toujours dans la démonstration du lemme 3.1). On distingue deux cas, selon la caractéristique de $F$ :

(a) $F$ est de caractéristique nulle.

LeMme 3.4. L'intégrale orbitale de $f^{\prime}$ s'annule sur les éléments elliptiques réguliers de $G$.

DÉmonstration. Soient $Z$ le centre de $G$ et $\omega$ un caractère unitaire de $Z$. Nous allons utiliser l'espace $L_{0}\left(G_{e} ; \omega\right)$ des fonctions $f$ localement constantes sur l'ensemble des éléments elliptiques réguliers de $G$, invariantes sous l'action de conjugaison par des éléments de $G$, et vérifiant $f(z g)=\omega(z) f(g)$ pour tout $z \in Z$ et tout $g$ elliptique régulier dans $G$. On 


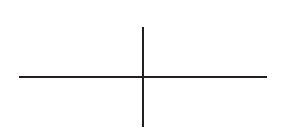

considère le sous-espace $L^{2}\left(G_{e} ; \omega\right)$ de $L_{0}\left(G_{e} ; \omega\right)$ formé des fonctions $f$ telles que

$$
\sum_{T \in \mathcal{T}_{e}}\left|W_{T}\right|^{-1} \int_{T^{\mathrm{reg}} / Z} D(\bar{t})|f(\bar{t})|^{2} d \bar{t}
$$

converge, où $\mathcal{T}_{e}$ est un ensemble de représentants des classes de conjugaison de tores elliptiques maximaux de $G,\left|W_{T}\right|$ est le cardinal du groupe de Weyl de $T, T^{\text {reg }}$ est l'ensemble des éléments réguliers de $T, d \bar{t}$ est choisie de façon à ce que le volume de $T^{\text {reg }} / Z$ soit 1 , et $D(\bar{t})$ est la valeur absolue normalisée du déterminant de l'opérateur $\operatorname{Ad}\left(\mathrm{t}^{-1}\right)-\operatorname{Id}$ agissant sur $\operatorname{Lie}(G) / \operatorname{Lie}(T)$. On définit un produit scalaire dans $L^{2}\left(G_{e} ; \omega\right)$ en posant:

$$
\left\langle f_{1} ; f_{2}\right\rangle_{e}=\sum_{T \in \mathcal{T}_{e}}\left|W_{T}\right|^{-1} \int_{T^{\mathrm{reg}} / Z} D(\bar{t}) f_{1}(\bar{t}) \overline{f_{2}(\bar{t})} d \bar{t},
$$

qui munit $L^{2}\left(G_{e} ; \omega\right)$ d'une structure d'espace préhilbertien.

On sait que pour toute représentation $\pi$ de $G$ de carré intégrable et de caractère central $\omega$, la restriction du caractère $\chi_{\pi}$ de $\pi$ à $G_{e}$ se trouve dans $L^{2}\left(G_{e} ; \omega\right)$ et les éléments de $L^{2}\left(G_{e} ; \omega\right)$ ainsi obtenus forment une famille orthonormale pour $\langle;\rangle_{e}([\mathrm{Cl}]$, on est en caractéristique nulle). Une conséquence de la correspondance de Jacquet-Langlands avec une algèbre à division est que ce système est complet ([Ba2], cor. 5.13, par exemple). À partir de $f^{\prime}$ on définit $f_{\omega}^{\prime}$ en posant, pour tout $g \in G$,

$$
f_{\omega}^{\prime}(g)=\int_{Z} \omega(z) f^{\prime}(z g) d z .
$$

Nous avons la relation entre les intégrales orbitales

$$
\Phi\left(f_{\omega}^{\prime} ; g\right)=\int_{Z} \omega(z) \Phi\left(f^{\prime} ; z g\right) d z,
$$

pour tout $g \in G$. Le lemme 3.3 entraîne donc que l'intégrale orbitale de $f_{\omega}^{\prime}$ est nulle sur les éléments semisimples réguliers qui ne sont pas elliptiques, i.e. qui n'appartiennent pas à un tore elliptique maximal. Dans ce cas, la formule d'intégration de Weyl donne, pour toute représentation de carré intégrable $\pi$ de $G$ de caractère central $\omega$,

$$
\operatorname{tr} \pi\left(f_{\omega}^{\prime}\right)=\sum_{T \in \mathcal{T}_{e}}|W(T)|^{-1} \int_{T^{\mathrm{reg}} / Z} D(\bar{t}) \chi_{\pi}(\bar{t}) \Phi\left(f_{\omega}^{\prime} ; \bar{t}\right) d \bar{t} .
$$

Or, on sait que $\operatorname{tr} \pi\left(f_{\omega}^{\prime}\right)=\operatorname{tr} \pi\left(f^{\prime}\right)=0$.

LEMME 3.5. La restriction à l'ensemble des éléments elliptiques réguliers de $G$ de l'intégrale orbitale de $f_{\omega}^{\prime}$ se trouve dans l'espace $L^{2}\left(G_{e} ; \omega^{-1}\right)$.

DÉmonstration. D'après [H-CvD], th.14, chap.8, pour tout $T$ dans $\mathcal{T}_{e}$, le produit de l'intégrale orbitale de $f_{\omega}^{\prime}$ et de la fonction $D^{1 / 2}$ est borné sur $T^{\text {reg }}$. Le module du carré de cette fonction est ainsi borné et donc intégrable sur $T^{\text {reg }} / Z$ qui est de mesure 1 . Comme $\mathcal{T}_{e}$ est fini, le résultat s'ensuit.

D'après ce qui précède, la fonction conjuguée complexe de $\Phi\left(f_{\omega}^{\prime} ; \cdot\right)$ est un élément de $L^{2}\left(G_{e} ; \omega\right)$ orthogonal à $\chi_{\pi}$. C'est vrai pour toute représentation $\pi$ de carré intégrable et de 


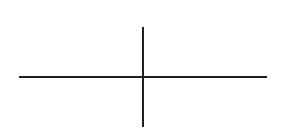

caractère central $\omega$. On en déduit que $\Phi\left(f_{\omega}^{\prime} ; \cdot\right)$ est identiquement nulle sur l'ensemble des éléments elliptiques réguliers.

Soit $g$ un élément elliptique régulier de $G$. Nous avons trouvé que pour tout caractère unitaire $\omega$ de $Z$ on a

$$
\int_{Z} \omega(z) \Phi\left(f^{\prime} ; z g\right) d z=0 .
$$

La proposition 4.4, [Bo], Chap. 2, implique que $\Phi\left(f^{\prime} ; g\right)=0$. Le lemme est montré.

Les lemmes 3.3 et 3.4 impliquent que l'intégrale orbitale de $f^{\prime}$ est nulle sur l'ensemble des éléments semisimples réguliers de $G$. Mais alors la trace de toute représentation de $G$ est nulle sur $f^{\prime}$ (nous sommes en caractéristique nulle). Cela contredit $\operatorname{tr}\left(\tau_{1}\left(f^{\prime}\right)\right) \neq 0$. En conclusion $s=1$ et on a $\operatorname{ind}_{P_{0}}^{G} \pi_{0}=a \tau$ où $a$ est un entier strictement positif et $\tau$ est une représentation tempérée de $G$.

(b) $F$ est de caractéristique positive. Nous avons utilisé deux résultats connus pour l'instant uniquement en caractéristique nulle dans la démonstration plus haut; le deuxième est le résultat de $[\mathrm{H}-\mathrm{CvD}]$ utilisé dans le lemme 3.5 , et le premier est l'intégrabilité locale des caractères, utilisée quand on a appliqué la formule d'intégration de Weyl (ce dernier résultat n'est pas connu en caractéristique non nulle, puisque [Ba3], bien que publié avant, se base sur le présent papier, désolé). Dans le cas de caractéristique non nulle, nous allons utiliser les corps proches de Kazhdan pour conclure. Soit $E$ un corps local non archimédien de caractéristique nulle. Notons $O_{F}$ (resp. $O_{E}$ ) l'anneau des entiers de $F$ (resp. $E$ ), et $I_{F}$ (resp. $I_{E}$ ) l'idéal maximal de $O_{F}$ (resp. $O_{E}$ ). Nous disons que $E$ et $F$ sont $m$-proches s'il existe un isomorphisme d'anneaux $\bar{\lambda}_{F E}^{m}$ de $O_{F} / I_{F}^{m}$ sur $O_{E} / I_{E}^{m}$ (pour tout $m$ on peut trouver un tel corps $E$ ). Quand nous dirons par la suite que $F$ et $E$ sont $m$-proches, on considérera tacitement qu'un isomorphisme $\bar{\lambda}_{F E}^{m}$ est fixé une fois pour toutes. Soit $E$ un corps $m$-proche de $F$. Rebaptisons $G_{F}$ notre groupe pour rappeler qu'il est défini sur $F$. Nous avons $G_{F}=$ $G L_{r}\left(D_{F}\right)$ où $D_{F}$ est une algèbre à division centrale sur $F$, de dimension finie $d^{2}$. On note $G_{E}$ le groupe $G L_{r}\left(D_{E}\right)$ où $D_{E}$ est une algèbre à division centrale sur $E$ qui a la même dimension et le même invariant (associé par la théorie du groupe de Brauer) que ceux de $D_{F}$.

Notons $O_{D_{F}}$ l'anneau des entiers de $D_{F}$, et $I_{D_{F}}$ l'idéal maximal de $O_{D_{F}}$. On pose $K_{D_{F}}^{0}=G L_{r}\left(O_{D_{F}}\right)$, et, pour tout entier strictement positif $l, K_{D_{F}}^{l}=1+M_{r}\left(I_{D_{F}}^{d l}\right)$. On fixe sur $G_{F}$ une mesure de Haar telle que le volume de $K_{D_{F}}^{0}$ soit 1 . Soit $f$ une fonction localement constante à support compact sur $G_{F}$. Nous définissons le niveau de $f$ comme étant le plus petit entier $l$ tel que $f$ soit bi-invariante par $K_{D_{F}}^{l}$. Notons $H_{F}^{l}$ l'algèbre de Hecke des fonctions localement constantes à support compact de niveau inférieur ou égal à $l$ sur $G_{F}$. Si $\pi$ est une représentation lisse irréductible de $G_{F}$ on appelle niveau de $\pi$ le plus petit entier $l$ tel que $\pi$ ait un vecteur non nul fixe sous $K_{D_{F}}^{l}$. Adoptons pour $G_{E}$ des notations et définitions analogues à celles fixées dans ce paragraphe pour $G_{F}$.

Dans [Ba2] nous montrons que, quel que soit l'entier positif $l$, il existe un entier $m$ tel que, pour tout corps $E m$-proche de $F, \bar{\lambda}_{F E}^{m}$ induise un isomorphisme d'algèbres $\bar{\zeta}_{D_{F} D_{E}}^{l}$ 


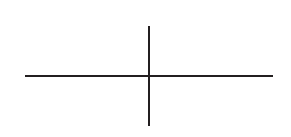

de $H_{F}^{l}$ sur $H_{E}^{l}$. D'oú une bijection entre l'ensemble de classes d'équivalence des représentations lisses irréductibles de $G_{F}$ de niveau inférieur ou égal à $l$ et l'ensemble de classes d'équivalence des représentations lisses irréductibles de $G_{E}$ de niveau inférieur ou égal à $l$. On utilise pour cet isomorphisme la même notation $\bar{\zeta}_{D_{F} D_{E}}^{l}$. Nous avons

$$
\operatorname{tr} \bar{\zeta}_{D_{F} D_{E}}^{l}(\pi)\left(\bar{\zeta}_{D_{F} D_{E}}^{l}(f)\right)=\operatorname{tr} \pi(f)
$$

pour toute représentation $\pi$ de $G_{F}$ de niveau inférieur ou égal à $l$ et toute $f \in H_{F}^{l}$.

Avec les conventions faites en début de section, un sous-groupe de Levi standard $L$ de $G_{F}$ ou $G_{E}$ est formé des matrices diagonales par blocs de taille donnée et on peut associer à $L$ de façon biunivoque une suite ordonnée d'entiers strictement positifs $r_{1}, r_{2}, \ldots, r_{k}$ telle que $\sum_{i=1}^{k} r_{i}=r$ où les $r_{i}$ représentent les tailles de ces blocs. À un sous-groupe de Levi standard de $G_{F}$ correspond donc un unique sous-groupe de Levi standard de $G_{E}$. C'est pareil pour les sous-groupes paraboliques standard. Si $L_{F}$ est un sous-groupe de Levi standard de $G_{F}$ on note $L_{E}$ le sous-groupe de Levi standard de $G_{E}$ qui lui correspond, et si $P_{F}$ est un sous-groupe parabolique standard de $G_{F}$ on note $P_{E}$ le sous-groupe parabolique standard de $G_{E}$ qui lui correspond. Sur chaque bloc d'un sous-groupe de Levi standard de $G_{F}$ et $G_{E}$ nous adoptons les mêmes notations et conventions que plus haut.

Soit $P$ est un sous-groupe parabolique standard de $G_{F}$ et $P=L U$ sa décomposition de Levi ( $L$ sous-groupe de Levi standard de $G_{F}$ et $U$ le radical unipotent de $P$ ). On munit $P$ et $U$ de mesures de Haar invariantes à gauche telles que les volumes de $P \cap K_{D_{F}}^{0}$ et $U \cap K_{D_{F}}^{0}$ soient égaux à 1 . On note $\delta_{P}$ le caractère modulaire sur $P$.

Pour toute fonction localement constante à support compact sur $G_{F}$, on définit une fonction localement constante à support compact sur $L, f^{P}$, par la formule:

$$
f^{P}(l)=\delta_{P}^{1 / 2}(l) \int_{K_{D_{F}}^{0}} \int_{U} f\left(k^{-1} l u k\right) d k d u
$$

pour tout $l \in L$.

Les mêmes définitions s'appliquent à $E$.

Proposition 3.6. Soient $P_{F}$ un sous-groupe parabolique standard de $G_{F}$ et $P_{F}=$ $L_{F} U_{F}$ la décomposition de Levi standard de $P_{F}$. Alors il existe un $m$ tel que, si $F$ et $L$ sont m-proches, alors $\bar{\zeta}_{D_{F} D_{E}}^{l}\left(f^{\prime}\right)$ et $\bar{\zeta}_{D_{F} D_{E}}^{l}\left(f^{\prime P_{F}}\right)$ sont bien définies et on a

$$
\left(\bar{\zeta}_{D_{F} D_{E}}^{l}\left(f^{\prime}\right)\right)^{P_{E}}=\bar{\zeta}_{D_{F} D_{E}}^{l}\left(f^{\prime P_{F}}\right) .
$$

DÉmonstration. L'analogue de cette proposition dans le cas particulier $G_{F}=$ $G L_{r}(F)$ est montré à la page 1053 de [Le]. La démonstration est exactement la même dans notre cas.

Nous allons relever notre situation en caractéristique nulle et utiliser (a). Soit $\mathcal{P}_{F}$ l'ensemble de tous les sous-groupes paraboliques standard, propres ou non, de $G_{F}$ et soit $m$ un entier suffisamment grand pour que, pour tout corps local $E$ qui est $m$-proche de $F$, la proposition 3.6 plus haut soit vérifiée pour tout $P_{F} \in \mathcal{P}_{F}$ qui a une décomposition de Levi standard $P_{F}=L_{F} U_{F}$ (c'est possible puisque $\mathcal{P}_{F}$ est un ensemble fini). On a alors: 


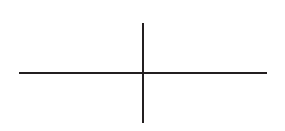

1) si $P_{E}$ est un sous-groupe parabolique standard propre de $G_{E}$, si $P_{E}=L_{E} U_{E}$ est une décomposition de Levi standard de $P_{E}$, si $\pi$ est une représentation lisse irréductible de $L_{E}$, alors:

- ou bien le niveau de $\pi$ est supérieur strictement à $l$ et alors

$$
\operatorname{tr} \pi\left(\bar{\zeta}_{D_{F} D_{E}}^{l}\left(f^{\prime P_{F}}\right)\right)=0
$$

- ou bien le niveau de $\pi$ est inférieur ou égal à $l$ et alors, en supposant que $\sigma$ est la représentation lisse irréductible de $L_{F}$ qui vérifie $\bar{\zeta}_{D_{F} D_{E}}^{l}(\sigma)=\pi$ on peut écrire:

$$
\operatorname{tr} \pi\left(\bar{\zeta}_{D_{F} D_{E}}^{l}\left(f^{\prime P_{F}}\right)\right)=\operatorname{tr} \sigma\left(f^{\prime P_{F}}\right)=\operatorname{tr}\left(\operatorname{ind}_{P_{F}}^{G_{F}} \sigma\right)\left(f^{\prime}\right)=0
$$

car $f^{\prime}$ annule la trace de toute représentation de $\operatorname{Grot}_{\text {ind }}\left(G_{F}\right)$.

Dans les deux cas on obtient $\operatorname{tr} \pi\left(\bar{\zeta}_{D_{F} D_{E}}^{l}\left(f^{\prime P_{F}}\right)\right)=0$ ce qui implique, compte tenu de la proposition 3.6 plus haut, que

$$
\operatorname{tr}\left(\operatorname{ind}_{P_{E}}^{G_{E}} \pi\right)\left(\bar{\zeta}_{D_{F} D_{E}}^{l}\left(f^{\prime}\right)\right)=0 .
$$

Finalement, on a trouvé que $\bar{\zeta}_{D_{F} D_{E}}^{l}\left(f^{\prime}\right)$ est une fonction qui annule la trace de toute représentation dans $\operatorname{Grot}_{\text {ind }}\left(G_{E}\right)$.

2) si $\pi$ est une représentation essentiellement de carré intégrable de $G_{E}$, alors ou bien son niveau est supérieur strictement à $l$ et donc $\operatorname{tr} \pi\left(\bar{\zeta}_{D_{F} D_{E}}^{l}\left(f^{\prime}\right)\right)=0$ ou bien son niveau est inférieur ou égal à $l$ et alors, en supposant que $\sigma$ est la représentation lisse irréductible de $G_{F}$ qui vérifie $\bar{\zeta}_{D_{F} D_{E}}^{l}(\sigma)=\pi$ on a que $\sigma$ est une représentation essentiellement de carré intégrable (th. 2.16.b dans [Ba2]) et par conséquent

$$
\operatorname{tr} \pi\left(\bar{\zeta}_{D_{F} D_{E}}^{l}\left(f^{\prime}\right)\right)=\operatorname{tr} \sigma\left(f^{\prime}\right)=0,
$$

car $f^{\prime}$ annule la trace de toute représentation essentiellement de carré intégrable de $G_{F}$. Finalement, on a trouvé que $\bar{\zeta}_{D_{F} D_{E}}^{l}\left(f^{\prime}\right)$ annule la trace de toute représentation essentiellement de carré intégrable de $G_{E}$.

Or, $E$ est de caractéristique nulle. Par les points 1) et 2) ci-dessus et par le raisonnement déjà fait en caractéristique nulle, on en déduit que $\bar{\zeta}_{D_{F} D_{E}}^{l}\left(f^{\prime}\right)$ annule la trace de toutes les représentations de $G_{E}$. D'autre part, comme $\operatorname{tr} \tau_{1}\left(f^{\prime}\right) \neq 0$, le niveau de $\tau_{1}$ est inférieur ou égal à $l$ et donc $\bar{\zeta}_{D_{F} D_{E}}^{l}\left(\tau_{1}\right)$ est bien défini et

$$
\operatorname{tr} \bar{\zeta}_{D_{F} D_{E}}^{l}\left(\tau_{1}\right)\left(\bar{\zeta}_{D_{F} D_{E}}^{l}\left(f^{\prime}\right)\right)=\operatorname{tr} \tau_{1}\left(f^{\prime}\right) \neq 0 .
$$

Contradiction! On trouve donc, comme en caractéristique nulle, que $s=1$ et on a $\operatorname{ind}_{P_{0}}^{G} \pi_{0}=$ $a \tau$ où $a$ est un entier strictement positif et $\tau$ est une représentation tempérée de $G$.

Le fait que $a=1$ se montre exactement de la même façon que les étapes (2) et (3) de la preuve de la proposition 27 de [FK], page 98. Le th. 1.1 est démontré.

\section{REFERENCES}

[Ba1] A. I. BADUlEsCU, Orthogonalité des caractères pour $G L_{n}$ sur un corps local de caractéristique non nulle, Manuscripta Math. 101 (2000), 49-70. 


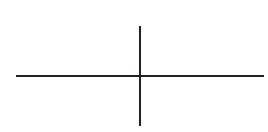

[Ba2] A. I. BADULESCU, Correspondance de Jacquet-Langlands en caractéristique non nulle, Ann. Sci. École Norm. Sup. (4) 35 (2002), 695-747.

[Ba3] A. I. BADULESCU, Un résultat de transfert et un résultat d'intégrabilité locale des caractères en caractéristique non nulle, à paraître dans J. Reine Angew. Math.

[BDK] J. Bernstein, P. Deligne And D. Kazhdan, Trace Paley-Wiener Theorem for reductive $p$-adic groups, J. Anal. Math. 47 (1986), 180-192.

[Bo] N. Bourbaki, Théories spectrales, Chap.1-2, Hermann, Paris, 1967.

[Cl] L. Clozel, Invariant harmonic analysis on the Schwartz space of a reductive p-adic group, ( Proc. Bowdoin Conf., 1989), 101-102, Progr. Math. 101, Birkhäuser, Boston, 1991.

[DKV] P. Deligne, D. KaZhDan And M.-F. VignéRAs, Représentations des algèbres centrales simples $p$ adiques, Représentations des groupes réductifs sur un corps local, Hermann, Paris, 1984.

[FK] Y. Flicker and D. KaZhdan, Metaplectic correspondence, Inst. Hautes Études Sci. Publ. Math. 64 (1986), 53-110.

[H-CvD] HARISH-ChAndRA, Harmonic Analysis on Reductive $p$-adic Groups, Notes by G. van Dijk, Lecture Notes in Math. 162, Springer-Verlag, Berlin-New York, 1970.

[Le] B. Lemaire, Intégrales orbitales sur $G L(N)$ et corps locaux proches, Ann. Inst. Fourier 46 (1996), $1027-1056$.

[Ta] M. TADIĆ, Induced representations of $G L(n ; A)$ for a $p$-adic division algebra $A$, J. Reine Angew. Math. 405 (1990), 48-77.

[Ze] A. ZelevinSKy, Induced representations of reductive $p$-adic groups II, Ann. Sci. École Norm. Sup. (4) 13 (1980), 165-210.

UNIVERSiTÉ DE POITIERS

UFR SCIENCES SP2MI

DÉPARTEMENT DE MATHÉMATIQUES

TÉLÉPORT 2 BP 30179

Boulevard Marie et Pierre Curie

86962 Futuroscope Chasseneuil Cedex

FRANCE

E-mail address: badulesc@math.univ-poitiers.fr 